\title{
Supporting Information: Interplay Between Optical Bianisotropy and Magnetism in Plasmonic
}

\section{Metamolecules}

Liuyang Sun ${ }^{1}$, Tzuhsuan Ma ${ }^{1}$, Seung-Cheol Yang ${ }^{1}$, Dong-Kwan Kim ${ }^{2}$, Gaehang Lee ${ }^{3}$, Jinwei Shi ${ }^{4}$, Irving Martinez ${ }^{5}$, Gi-Ra Yi ${ }^{2}$, Gennady Shvets ${ }^{1,6 *}$, and Xiaoqin $\mathrm{Li}^{1,6 *}$

1 Department of Physics and the Center for Complex Quantum Systems, The University of Texas at Austin, Austin, Texas 78712, United States

2 School of Chemical Engineering, Sungkyunkwan University, Suwon 16419, Republic of Korea

3 Korea Basic Science Institute and University of Science and Technology, Daejeon 34113, Republic of Korea

4 Department of Physics and Applied Optics Beijing Area Major Laboratory, Beijing Normal University, Beijing 100875, China

5 Department of Physics, University of Texas at El Paso, El Paso, Texas 79968, United States

6 Materials Science and Engineering Program, Texas Materials Institute, The University of Texas at Austin, Austin, Texas 78712, United States 


\section{Fitting of scattering spectrum at normal incident and calculation of $\kappa_{M} / \kappa_{E}$}

The normal incidence spectrum with $\phi=90^{\circ}$ is fitted with the following equation:

$$
\frac{S(0, \omega)}{S\left(0, \omega_{p}\right)}=\frac{\chi^{4}+2 \gamma_{\mu} \gamma_{p} \chi^{2}+\gamma_{p}^{2}\left[\gamma_{\mu}^{2}+\left(\omega_{p}-\omega_{\mu}\right)^{2}\right]}{\chi^{4}+2 \chi^{2}\left[\gamma_{\mu} \gamma_{p}-\left(\omega-\omega_{\mu}\right)\left(\omega-\omega_{p}\right)\right]+\left[\gamma_{\mu}^{2}+\left(\omega-\omega_{\mu}\right)^{2}\right]\left[\gamma_{p}^{2}+\left(\omega-\omega_{p}\right)^{2}\right]} \cdot \frac{\left(\omega-\omega_{\mu}\right)^{2}+\gamma_{\mu}^{2}}{\left(\omega_{p}-\omega_{\mu}\right)^{2}+\gamma_{\mu}^{2}}
$$

The scattering spectrum is normalized to the peak value at electric dipole resonance near $675 \mathrm{~nm}$.

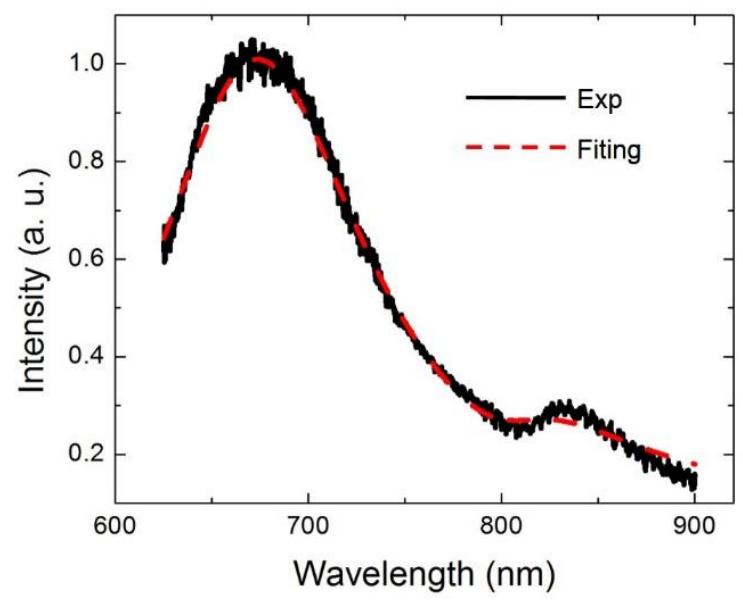

Figure S1. Fitting $S(0, \omega)$ to experimental data

Figure S1 shows the result of fitting. The extracted data are shown in Table S1.

Table S1, Summarized Fitting parameters

\begin{tabular}{|c|c|}
\hline$\omega_{p}$ & $2 \pi c \cdot(680.3 \mathrm{~nm})^{-1}$ \\
\hline$\omega_{\mu}$ & $2 \pi c \cdot(805.2 \mathrm{~nm})^{-1}$ \\
\hline$\gamma_{p}$ & $0.107 \omega_{p}$ \\
\hline$\gamma_{\mu}$ & $0.045 \omega_{m}$ \\
\hline
\end{tabular}




\begin{tabular}{|l|l|}
\hline$\chi$ & $0.0363 \omega_{p}$ \\
\hline
\end{tabular}

To solve $\kappa_{M} / \kappa_{E}$, we take the fitting results: $\omega_{(p) \mu}, \gamma_{(p) \mu}$ and $\chi$ as known parameters and algebraic solve Eq.(S2) with the $\frac{\Delta S\left(\theta, \omega_{\mu}\right)}{S\left(\theta, \omega_{p}\right)}=0.12$ extracted from the experiment results with oblique incidence at $\theta=60^{\circ}$.

$$
\frac{\Delta S\left(\theta, \omega_{\mu}\right)}{S\left(\theta, \omega_{p}\right)}=\left(\frac{\kappa_{M}}{\kappa_{E}}\right) \frac{4\left(\frac{\chi}{\gamma_{\mu}}\right) \sin \theta}{1+\left(\frac{\omega_{p}-\omega_{\mu}}{\gamma_{\mu}}\right)^{2}} \cdot \frac{\chi^{4}+2 \gamma_{\mu} \gamma_{p} \chi^{2}+\gamma_{p}^{2}\left[\gamma_{\mu}^{2}+\left(\omega_{p}-\omega_{\mu}\right)^{2}\right]}{\chi^{4}+2 \gamma_{\mu} \gamma_{p} \chi^{2}+\gamma_{\mu}^{2}\left[\gamma_{p}^{2}+\left(\omega_{\mu}-\omega_{p}\right)^{2}\right]}
$$

Note we have assumed $\kappa_{E}^{2}=\gamma_{p} \lesssim \gamma_{p, R}$ in the above calculation. This assumption is validated by comparing simulated scattering cross section (SCS) and absorption cross section (ACS) in Figure S2. A larger SCS in comparison to ACS confirms that radiation loss dominates over resistive loss.

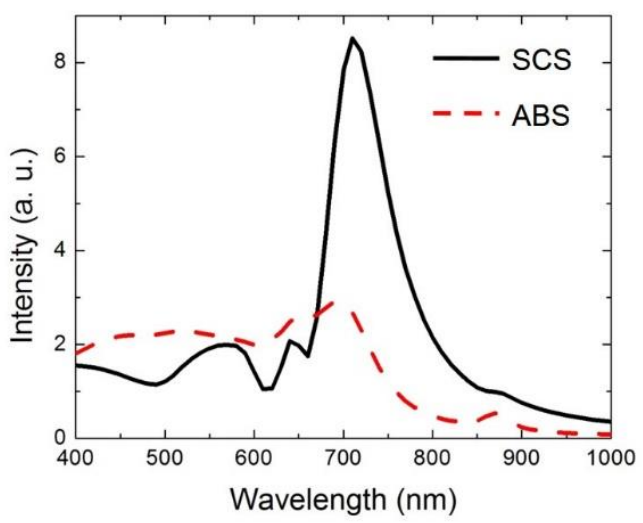

Figure S2. Calculated scattering and absorption cross section. 
This simulated normalized cross section spectra support the argument that $\kappa_{E}^{2} \lesssim \gamma_{p}$ in which $\frac{\kappa_{E}^{2}}{\gamma_{p}} \approx \frac{8.5}{8.5+2.9} \approx 0.75$ is based on the values at $710 \mathrm{~nm}$.

\section{Gap sizes used in the simulation}

In numerical simulations, we started from investigating the gap sizes $\left[g_{T}, g_{L}, g_{B}, g_{R}\right]=$ [g0,g0+dg, g0, g0-dg] where $\mathrm{g} 0=1(\mathrm{~nm})$ and $\mathrm{dg}=15 \% \sim 30 \%$ to $\mathrm{g} 0=0.5(\mathrm{~nm})$ and $\mathrm{dg}=15 \% \sim 30 \%$. During the process of decreasing size of gaps, the magnetic resonance peak red shifts if dg is kept the same in percentage. We found one set of parameters which produced simulated spectra qualitatively agreeing with the experimental results and presented the simulation results in the main text. We did not search for a wide parameter space numerically to obtain the best match with the experimental data because the main focus of the paper is to use the analytical solutions to the coupled mode equations and to capture the key spectral feature of asymmetric scattering due to MM and ME coupling.

Furthermore, we note that the gap size used in the simulation, $\sim 0.5 \mathrm{~nm}$, should be considered as an effective gap size instead of a precise parameter that represents the experimentally assembled structure. There are some experimental details not included in the simulation. For example, there are ligand molecules on the nanoparticle surfaces, which change the effective index of the gap. We only aim to find qualitative agreement between the numerical simulation and the experiments. 